\title{
Wide-View and Broadband Circular Polarizers for Transflective Liquid Crystal Displays
}

\author{
Zhibing Ge, Member, IEEE, Meizi Jiao, Ruibo Lu, Thomas X. Wu, Senior Member, IEEE, \\ Shin-Tson Wu, Fellow, IEEE, Wang-Yang Li, and Chung-Kuang Wei
}

\begin{abstract}
A simple wide-view and broadband circular polarizer comprising of a linear polarizer and two uniaxial films is proposed to enhance the viewing angle of transflective liquid crystal displays (LCDs). For the transmissive mode, over the entire $90^{\circ}$ viewing cone, the normalized light leakage from two stacked circular polarizers is suppressed to below $1.5 \times 10^{-2}$, and contrast ratio over 10:1 is obtained using a normally black vertically aligned transflective LCD. At the same time, this configuration warrants a broadband operation and reasonably good viewing angle (10:1 contrast ratio is over $40^{\circ}$ at all directions) for the reflective mode. The physical mechanisms for achieving broadband operation and wide viewing angle are discussed.
\end{abstract}

Index Terms-Broadband, circular polarizer, liquid crystal display (LCD), transflective, wide-viewing angle.

\section{INTRODUCTION}

$\mathbf{T}$ HE rapid emerging of mobile devices, such as cell phones and personal digital assistants, drives a heated research in developing transflective liquid crystal displays (LCDs) with high brightness and wide viewing angle. The optical efficiency of transflective LCDs has been continuously improved by developing optimized cell structures and driving schemes [1]-[7]. Yet, the viewing angle of most transflective LCDs is still fairly narrow because of the use of two crossed broadband circular polarizers (CPs). Although the recent progress in developing in-plane switching based transflective LCDs enhances the viewing angle of the transmissive mode, the challenge is its need for an in-cell phase retarder to assure a good dark state for the reflective mode [7]. On the other hand, it is very easy to obtain a common dark state for the transmissive $(\mathrm{T})$ and reflective $(\mathrm{R})$ modes in multi-domain VA LCD [8], but more researches are needed to improve its performance under circular polarizers.

A conventional cost-effective broadband circular polarizer consists of a linear polarizer, a uniaxial monochromatic half-wave plate, and a uniaxial monochromatic quarter-wave plate, with their optic axes aligned at certain angles [9], [10]. To reduce cost, the employed retardation films are usually made

Manuscript received September 26, 2007; revised November 29, 2007. This work was supported by Chi-Mei Optoelectronics Corporation, Tainan, Taiwan, R.O.C.

Z. Ge, M. Jiao, R. Lu, and S.-T. Wu are with the College of Optics and Photonics/CREOL, University of Central Florida, Orlando, FL 32816 USA (e-mail: zge@mail.ucf.edu).

T. X. Wu are with the School of Electrical Engineering and Computer Science, University of Central Florida, Orlando, FL 32816 USA.

W.-Y. Li and C.-K. Wei are with Chi-Mei Optoelectronics, Tainan, Taiwan 74144, R.O.C.

Digital Object Identifier 10.1109/JDT.2008.920178 of uniaxial positive A-plates. The produced polarization is circular only at normal incidence, while its off-axis light leakage is still quite evident. Replacing the uniaxial quarter-wave plate with a biaxial quarter-wave plate only slightly improves the acceptance angle [11], [12]. Optimizing both half-wave film and quarter-wave film by the combinations of uniaxial A-plates and C-plates [13] or two biaxial films [14] significantly widen the acceptance angle, but their device configurations are quite sophisticated. Recently, circular polarizers with two linear polarizers and a positive and a negative monochromatic quarter-wave A-plates are proposed for transmissive LCDs [15]. This configuration shows an excellent viewing angle for the transmissive mode, but has a limited bandwidth if applied for the reflective mode, owing to the fact that the top circular polarizer comprises a linear polarizer and a single monochromatic quarter-wave plate that is optimized only at one single wavelength (more details will be discussed later in this paper). Although another A-plate is inserted between the linear polarizer and the quarter-wave plate, it is not a half-wave plate and its optic axis is either perpendicular to or parallel with the absorption axis of the linear polarizer to compensate the polarizer effective angle deviation. Therefore, there is an urgent need to develop a simple low-cost circular polarizer for transflective LCDs with both wide viewing angle for transmissive mode (which is the major function used in most transflective LCDs and requires high performance) and broadband operation and reasonably good viewing angle for reflective mode (which is mainly used for information reading and more tolerable in viewing angle with the existence of small surface reflections).

In this paper, we propose a cost-effective, wide-view, and broadband circular polarizer configuration for transflective LCDs, where two circular polarizers can compensate with each other. In this configuration, each circular polarizer consists of a linear polarizer, a monochromatic uniaxial half-wave A-plate, and a monochromatic uniaxial quarter-wave A-plate, but the two half-wave plates (and two quarter-wave plates) from different circular polarizers are made of uniaxial A-films with opposite optical birefringence. Through optimization with the assistance of the Poincare sphere [16], [17], the off-axis phase retardation is well self-compensated and light leakage is greatly suppressed. Over the entire $90^{\circ}$ viewing cone, for the transmissive mode, the light leakage of the two stacked circular polarizers is less than $1.5 \times 10^{-2}$ (normalized to the maximum transmission from two parallel linear polarizers) and contrast ratio is over 10:1 using a multi-domain vertically aligned (MVA) LC cell. At the same time, for the reflective mode, a broadband operation is obtained and a contrast of 10:1 is obtained over $40^{\circ}$ at all viewing directions. 


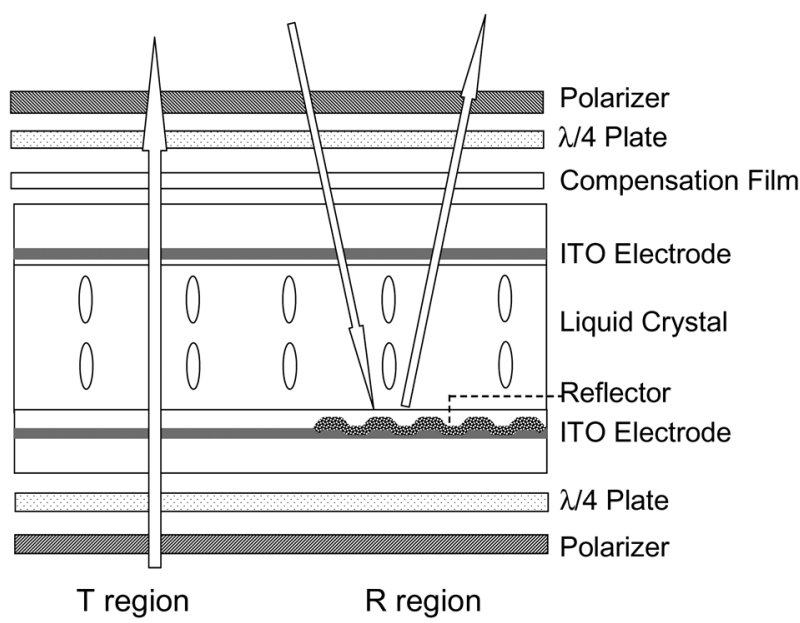

Fig. 1. Schematic structure of typical transflective LCD under circular polarizers.

\section{Design CONCEPT OF Wide-VIEW Circular Polarizers}

In our analysis, we trace the polarization state of the incident light on the Poincaré sphere by solving the electric fields using the 2-by-2 matrix method [18], [19] to get optimized results. The absorptive linear polarizer is modeled as a lossy uniaxial material. The phase retardation of the protective Tri-Acetyl-Cellulose (TAC) films is ignored. If not negligible, we can always add a positive $C$ plate to compensate the phase of the negative $\mathrm{C}$ plate from the TAC film. The refractive indices of the liner polarizer and the uniaxial retardation films are listed as follows: $n_{e, p o l}=1.5+i \times 2.208 \times 10^{-3}, n_{o, p o l}=1.5+i \times 3.222 \times 10^{-5}$, $n_{e, p A}=1.5902, n_{o, p A}=1.5866, n_{e, n A}=1.50$, and $n_{o, n A}=$ 1.60 (at wavelength $\lambda=550 \mathrm{~nm}$ ). Also, the birefringence dispersion of uniaxial material is characterized by the extended Cauchy equations [20]

$$
n_{e, o}=A_{e, o}+\frac{B_{e, o}}{\lambda^{2}}+\frac{C_{e, o}}{\lambda^{4}} .
$$

Fig. 1 depicts the cell structure of a typical transflective LCD, where each pixel is divided into a sub- $\mathrm{T}$ and a sub- $\mathrm{R}$ region. To achieve a dark state for the reflective mode, a circular polarizer placed above the LC cell is necessary [21], which further requires a second circular polarizer be placed below the LC cell to achieve a common dark state between the T- and R-modes. Here the circular polarizer can be realized by a linear polarizer and a monochromatic quarter-wave plate (narrowband design where the quarter-wave plate is aligned at $45^{\circ}$ away from the transmission axis of its adjacent linear polarizer) or by a linear polarizer with a broadband quarter-wave plate (broadband design).

Fig. 2(a) depicts the detailed configuration of the top and bottom conventional broadband circular polarizers; each consists of a linear polarizer and two uniaxial positive birefringence A-films $\left(n_{x}>n_{y}=n_{z}\right)$. Once the following condition is satisfied, the circular polarizer is a broadband device [9], [10]:

$$
2 \varphi_{\lambda / 4}-4 \varphi_{\lambda / 2}=90^{\circ}
$$

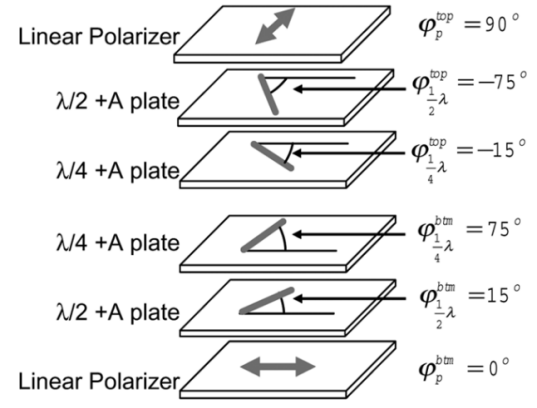

(a)

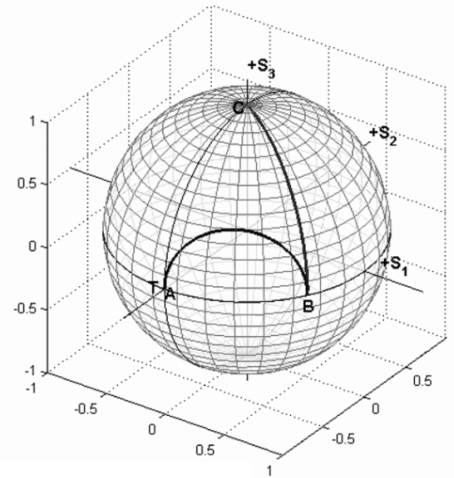

(b)

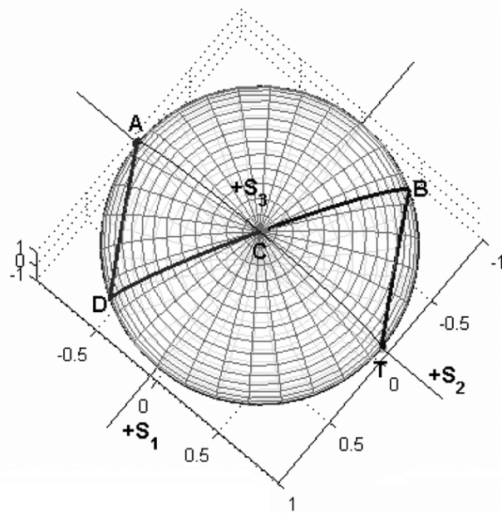

(c)

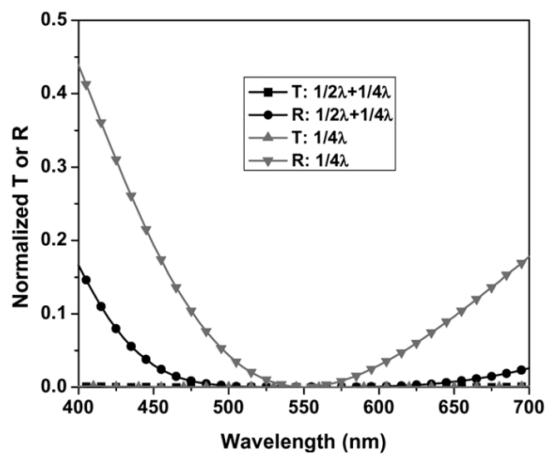

(d)

Fig. 2. (a) Configuration of crossed conventional broadband circular polarizers; polarization trace on the Poincare sphere at $\theta_{\text {inc }}=0^{\circ}$ for (b) the T-mode and (c) the R-mode; and spectral light leakages from conventional circular polarizers using one or two retardation plates.

where $\varphi_{\lambda / 2}$ is the azimuthal angle between the optic axis of the half-wave plate the transmission axis of the linear polarizer, and $\varphi_{\lambda / 4}$ is for the quarter-wave plate. If the transmission axis of 
a linear polarizer is along the $x$-axis, then $\varphi_{\lambda / 2}$ and $\varphi_{\lambda / 4}$ are chosen to be $15^{\circ}$ and $75^{\circ}$, respectively, as shown in Fig. 2(a) to minimize the light leakage at different wavelengths.

Fig. 2(b) depicts the polarization state trace on the Poincaré sphere when a light is incident to the transmissive structure from the bottom side at a normal direction. On the Poincare sphere, the transmission axis of the bottom polarizer is represented by the point $\mathbf{T}$, while point $\mathbf{A}$ represents the absorption axis of the top polarizer. Points $\mathbf{T}$ and $\mathbf{A}$ overlap with each other when the top and bottom polarizers are crossed. The incident light from the bottom polarizer is first rotated $30^{\circ}$ from point $\mathbf{T}$ to another linear polarization state at point $\mathbf{B}$ by the half-wave plate, then it is converted by the quarter-wave plate to a circular polarization on the north pole at point $\mathbf{C}$. This circularly polarized light at point $\mathbf{C}$ will be converted back to a linear polarization state at point $\mathbf{A}$ after traversing the top quarter-wave and half-wave plates, thus is blocked by the top linear polarizer, resulting in a dark state (their traces are overlapped with the previous lines). Here, $\varphi_{\lambda / 2} \sim 15^{\circ}$ is an optimal angle for minimizing the light leakage of a broadband light source, under which the $\operatorname{arcs} \overline{T B}$ and $\overline{B C}$ can compensate with each other for the wavelength variation in Fig. 2(b).

Fig. 2(c) shows the corresponding polarization trace for the reflective mode on the Poincare sphere, where the light comes from the ambient and is reflected back to the viewer by the metal reflector (not shown here) just below the top circular polarizer in Fig. 2(a). For the reflective mode, we can view the reflective structure as a transmissive structure by taking the reflective portion as a mirror image [19] to the incident one. In other words, a reflective display can be viewed as a transmissive structure like that in Fig. 2(a) but the bottom circular polarizer is replaced with the image of the top one, where a major difference from Fig. 2(a) is the corresponding wave plates (e.g., two half-wave plates) in the bottom and top parts have the same optic axis alignment. Under such a condition, the reflective mode works like a transmissive mode under parallel linear polarizers. Therefore, the incident light from the ambient first has its polarization at point $\mathbf{T}$ after passing the top linear polarizer, and the final absorption direction will be at point $\mathbf{A}$, where these two polarization directions are perpendicular to each other (point $\mathbf{T}$ and point $\mathbf{A}$ are $180^{\circ}$ away on the Poincaré sphere). The incident light from point $\mathbf{T}$ will be further converted to a circular polarization at point $\mathbf{C}$ by the top circular polarizer; after reflection on the reflector surface, the light is further converted to another linear polarization at point $\mathbf{A}$ by the mirror image part of the top circular polarizer, thus a good dark state also can be obtained. Here although on the metal reflector surface, the absolute handiness of the incident and exit lights is opposite, but under the mirror image method, the propagation direction of the exit light is also inverted, so the exit circularly polarized light can still be represented on the point $\mathbf{C}$ from Fig. 2(c) under one transmissive structure.

Fig. 2(d) plots the spectral light leakages (transmittance or reflectance) from both the broadband circular polarizer structure in Fig. 2(a) and the narrow band structure comprising a linear polarizer and a $45^{\circ}$ aligned monochromatic quarter-wave plate. In the calculations here, the spectral transmittance from two parallel linear polarizers (the real absorption coefficients of linear polarizers at all wavelengths are taken into consideration) at normal incidence is calculated first as a reference value, and the spectral light leakages obtained later are normalized to this reference transmittance. It can be seen that the transmittance from both crossed narrowband and broadband circular polarizers is small, which mainly results from the crossed structure between two corresponding wave plates (e.g., top and bottom half-wave plates, or top and bottom quarter-wave plates) under crossed polarizers that can compensate for wavelength dispersion at normal incidence. As for the reflective mode, it can be viewed like a transmissive structure with the upper circular polarizer and its mirror image below. Therefore, the self-compensation coming from the crossed structure as in the transmissive mode no longer exists. But for the reflective mode in the broadband structure, the compensation between the half-wave plate and quarter-wave plate within each circular polarizer works to warrant a broadband operation. The effect is still not as good as the transmissive mode [as we can see in the shorter wavelength region in Fig. 2(d)]. For the narrowband circular polarizer with a single quarter-wave plate, the light leakage is even severer and a good dark state is only warranted at the designed wavelength.

However, the transmissive mode even in the broadband circular polarizers has a large off-axis light leakage. At an oblique incident angle, the phase retardations from all these same typed positive birefringence wave plates will accumulate to make the final polarization deviate far away from the absorption direction of the top circular polarizers, resulting in light leakage. Fig. 3(a) shows the corresponding polarization state trace from Fig. 2(a) when incident angle $\theta_{\text {inc }}$ is equal to $40^{\circ}$. The final polarization state at point $\mathbf{F}$ just before reaching the top linear polarizer departs far away from the absorption direction at point $\mathbf{A}$, yielding an incomplete absorption of the light. Fig. 3(b) plots the angular light leakage for transmissive structure, respectively, where it is normalized to the reference transmittance from two parallel linear polarizers at normal incidence. Here, the light leakage at $\theta_{\text {inc }}=40^{\circ}$ is about $10 \%$, which also indicates the viewing cone with contrast ratio over $10: 1$ is limited to $40^{\circ}$. This is not enough for today's high performance transflective LCDs requiring wide-viewing angle. Here, for the transmissive mode, the severe light leakages are attributed to two sources: 1) the disparity between the transmission axis of the bottom polarizer (point $\mathbf{T}$ ) and the absorption axis of the top polarizer (point A) when viewed from an oblique direction and 2) the off-axis phase retardation accumulation from the four positive A-plates. Similar results with large light leakages can be obtained for the reflective mode as illustrated in Fig. 3(c) and (d).

To minimize light leakage at oblique angles, we propose here to use opposite birefringence materials for the two half-wave and two quarter-wave plates. These positive and negative birefringence films will self-compensate the off-axis phase retardations and thus make the final polarization closer to the absorption axis. In reference to Fig. 2(a), different film combinations can be used from bottom to top, but they can be grouped into two categories: 1) same-typed wave plates are employed within each circular polarizer, e.g., the bottom circular polarizer uses both positive (or negative) A-plates, and both negative (or positive) A-plates are employed in the top circular polarizer and 2) opposite-typed wave plates are employed within each circular polarizer, e.g., the bottom circular polarizer uses one positive (or negative) A-plate as its half-wave plate and one negative (or 


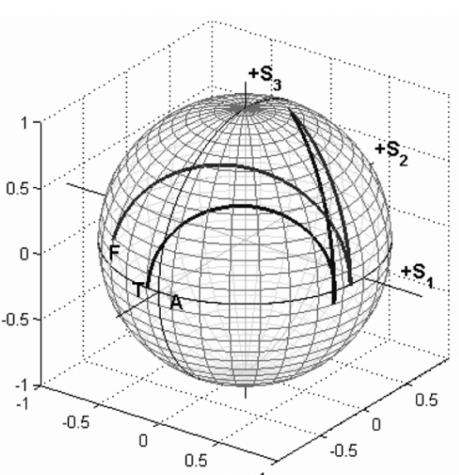

(a)

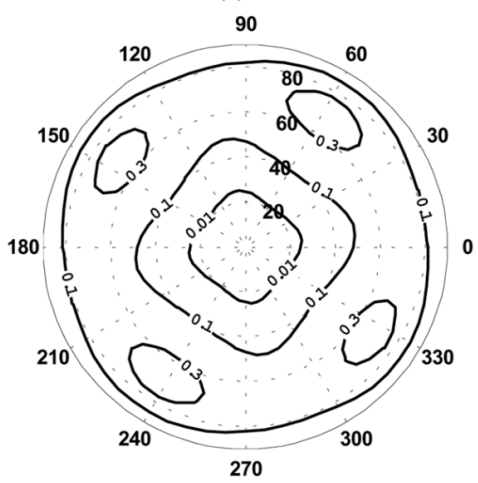

(b)

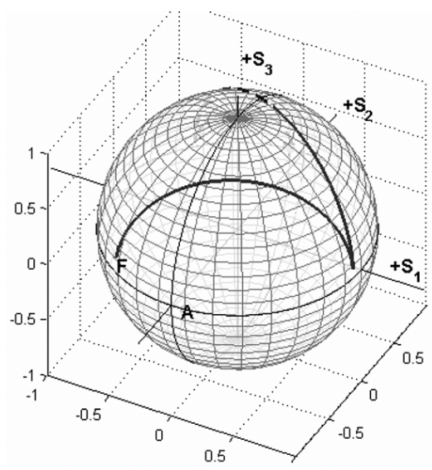

(c)

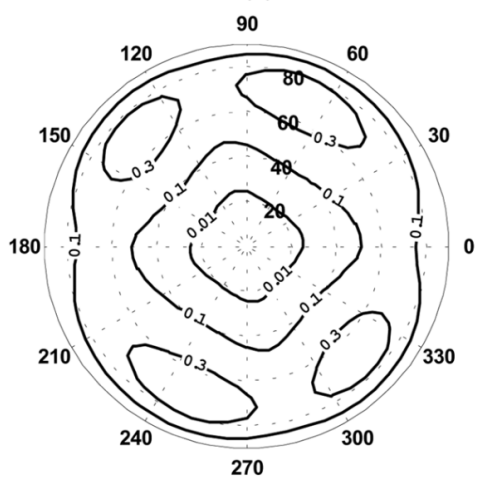

(d)

Fig. 3. (a) Polarization trace when viewed at $\theta_{\text {inc }}=40^{\circ}$ and $\varphi_{\text {inc }}=-45^{\circ}$ for the T-mode and (b) its angular light leakage; (c) polarization trace when viewed at $\theta_{\text {inc }}=40^{\circ}$ and $\varphi_{\text {inc }}=-45^{\circ}$ for the R-mode; and (d) its angular light leakage.

positive) A-plate as its quarter-wave plate, and the top circular polarizer correspondingly uses one negative (or positive) A plate as the half-wave plate and one positive (or negative) A plate as the quarter-wave plate.

To maintain the wide-view and broadband properties, three major requirements determine the orientation of their optic axes among these retardation films: 1) to compensate the off-axis phase retardation between two half-wave plates (or two quarterwave plates) with opposite sign of birefringence, their optic axes need to be aligned close to each other; 2) to achieve broadband properties of each circular polarizer, the optic axis orientation of each half-wave plate and its adjacent quarter-wave plate need to satisfy a similar relationship to (2), as to be discussed later; and 3 ) the top circular polarizer must be broadband to guarantee the spectral performance of the reflective mode, i.e., the angle between the optic axis of the top half-wave plate and the top polarizer transmission axis needs to be close to $\pm 15^{\circ}$ (or $\pm 75^{\circ}$ ). In Section II-A and II-B, we will use the Poincaré sphere to derive their angular relations according to different film combinations. For convenience, the transmission axis of the bottom linear polarizer is set along $x$-axis, and the top polarizer is always crossed to the bottom one. Here the discussion is based on the two main categories of wave plate combinations and the angle between the optic axis of the top half-wave plate and the top linear polarizer is taken to be close to $\pm 15^{\circ}$.

\section{A. Same Typed Wave Plates Within Each Circular Polarizer}

Fig. 4(a) shows the film configuration of a wide-view and broadband circular polarizer, where the top half-wave plate is a negative A-plate with its optic axis aligned at $\varphi_{\lambda / 2}^{\mathrm{btm}}=75^{\circ}$ with respect to the $x$-axis, which is also $-15^{\circ}$ away from the transmission axis of the top polarizer. Accordingly, the angle $\varphi_{\lambda / 2}^{\mathrm{btm}}$ of the bottom half-wave positive A-plate is at $\sim 75^{\circ}$. The orientation of the optic axis of the quarter-wave plate can be determined with assistance of the Poincaré sphere.

Fig. 4(b) shows the polarization state traced on the Poincaré sphere at normal incidence, where the transmission axis of the bottom polarizer (point $\mathbf{T}$ ) overlaps with the absorption direction (point A) of the top polarizer. On the Poincaré sphere, the optic axes of the bottom half-wave plate and quarter-wave plate are at $2 \varphi_{\lambda / 2}^{\mathrm{btm}}$ and $2 \varphi_{\lambda / 1}^{\mathrm{btm}}$ with respect to the axis $\mathbf{O T}$ on the equator [16], [17]. The linearly polarized light from the bottom polarizer is first rotated by the bottom half-wave plate to point $\mathrm{B}$ by $4 \varphi_{\lambda / 2}\left(\angle B O T=300^{\circ}\right)$. In order to assure the broadband property, the following quarter-wave plate made of a positive A-film must transfer the polarization from point $\mathbf{B}$ to the north pole $\mathbf{C}$ into the upper semi-sphere, making the arc $\overline{T B}$ and arc $\overline{B C}$ in the same semi-sphere to compensate each other for the wavelength dispersion. From the geometry relations on the equator, the optic axis of the bottom quarter-wave positive A-plate at $2 \varphi_{\lambda / 4}^{\mathrm{btm}}$ needs to be $90^{\circ}$ ahead of the axis $\mathbf{O B}$ at $4 \varphi_{\lambda / 2}^{\mathrm{btm}}$. Thus, a general relation between these angles is derived as follows:

$$
2 \varphi_{(1 / 4) \lambda}-4 \varphi_{(1 / 2) \lambda}=90^{\circ}+2 m \pi
$$

where $m$ is an integer which could be 0 , and \pm 1 . For the angles plotted in Fig. 4(a), $m=-1$. The circularly polarized light at point $\mathbf{C}$ is transferred back to point $\mathbf{A}$ by the top half-wave and 


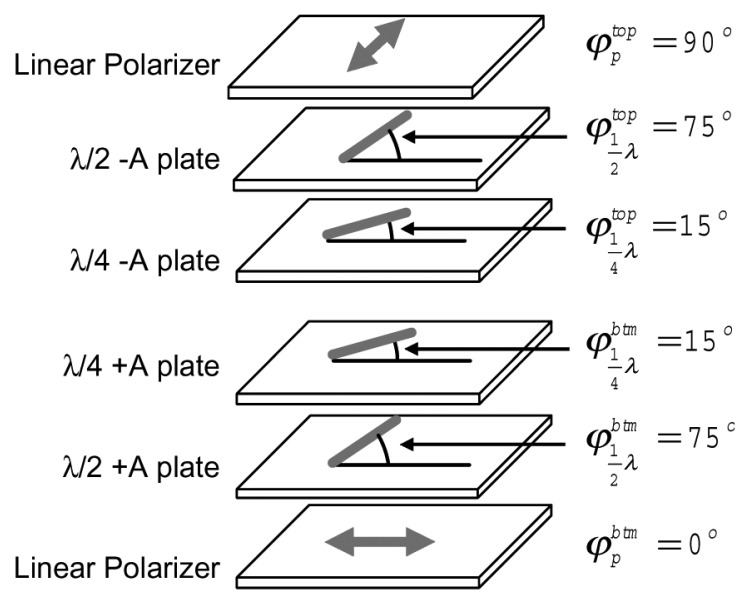

(a)

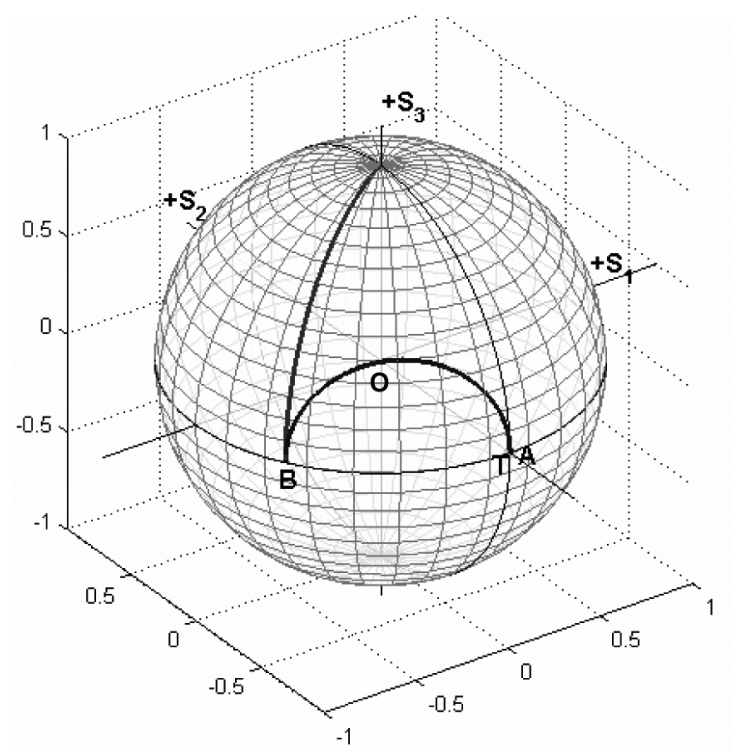

(b)

Fig. 4. (a) Crossed wide-view and broadband circular polarizers using positive and negative A-plates and (b) its polarization trace when viewed at $\theta_{\text {inc }}=0^{\circ}$.

quarter-wave plates, and blocked by the top linear polarizer. As a result, a good dark state is obtained.

On the other hand, if $\varphi_{\lambda / 2}^{\text {top }}=-75^{\circ}$, or the optic axis of the top half-wave plate $+15^{\circ}$ ahead of the transmission axis of the top polarizer, as shown in Fig. 5(a). The general relation between the optic axes of the half-wave positive A-plate and quarter-wave positive A-plate within each circular polarizer needs to satisfy the following relation:

$$
2 \varphi_{(1 / 4) \lambda}-4 \varphi_{(1 / 2) \lambda}=-90^{\circ}+2 m \pi
$$

where $m$ is an integer which could be 0 and \pm 1 . In Fig. 5(a) we show the films configuration for $m=1$. In this case, all the traces are confined to the bottom semi-sphere, as shown in Fig. 5(b) to guarantee a broadband operation.

\section{B. Opposite-Typed Wave Plates Within Each Circular Polarizer}

Fig. 6(a) and (b) shows another two possible configurations for the wide-view and broadband circular polarizers with alter-

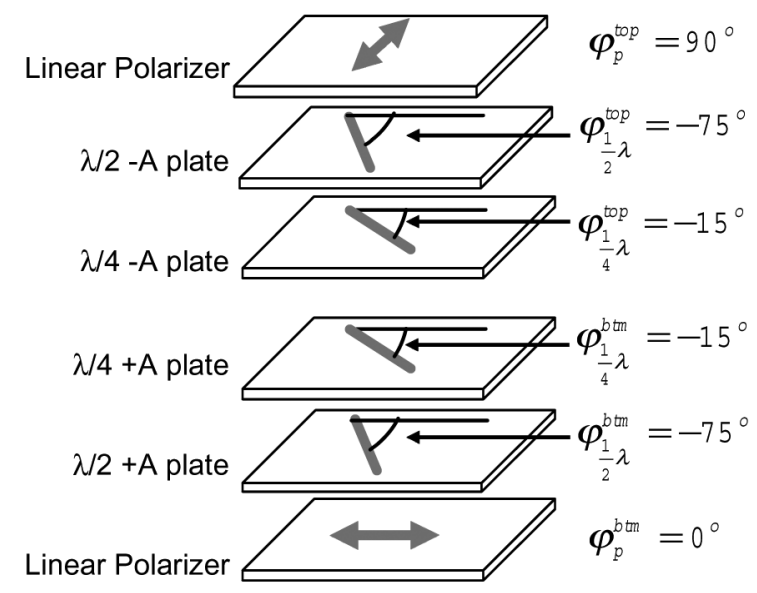

(a)

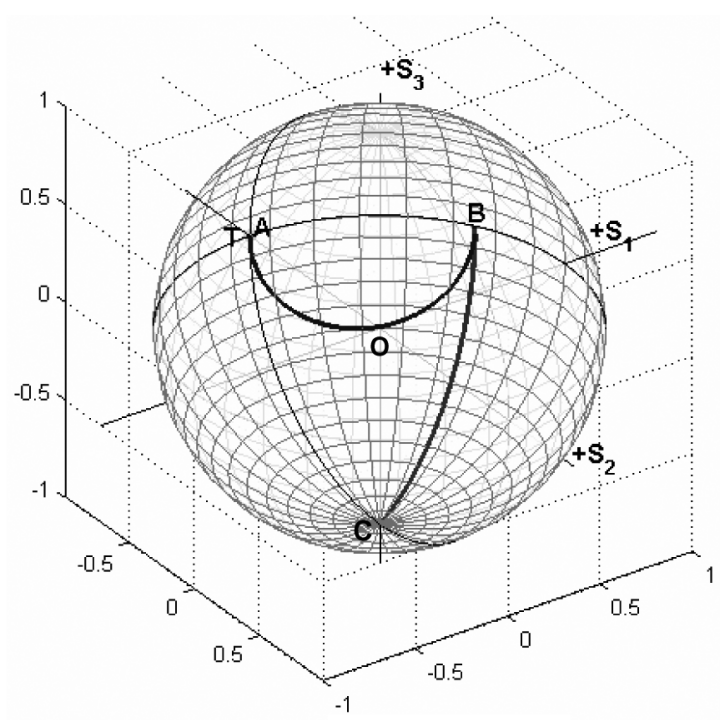

(b)

Fig. 5. (a) Film configuration of two crossed wide-view and broadband circular polarizers and (b) its polarization trace when viewed at $\theta_{\text {inc }}=0^{\circ}$.

nating positive/negative uniaxial A-plates. To maintain broadband in the entire visible spectral region, when the optic axis of the top half-wave plate is at $-15^{\circ}$ from the transmission axis of the top polarizer, i.e., $\varphi_{\lambda / 2}^{t o p}=75^{\circ}$ with respect to the $x$-axis, the optic axes of the half-wave plate and quarter-wave plate need to satisfy (4). On the other hand, these axes need to satisfy (3) when the top half-wave plate has its optic axis aligned at $\varphi_{\lambda / 2}^{\mathrm{btm}}=-75^{\circ}$ to the $x$-axis, which is also $+15^{\circ}$ ahead of the transmission axis of the top polarizer.

Although there are several configurations, the two in Fig. 6(a) and (b) would have better off-axis performance for the reflective mode than those in Figs. 4(a) and 5(a), because self-compensation from the opposite sign of birefringence between the two wave plates also exist in the top circular polarizer. Therefore, in Section III only the configuration in Fig. 6(a) will be used as examples for illustrating our design principles. Other designs including the configurations shown in Figs. 4(a)-6(b) also work as broadband and wide-view circular polarizers for a transflective LCD. However, their analysis is similar so that their results are not included here. 


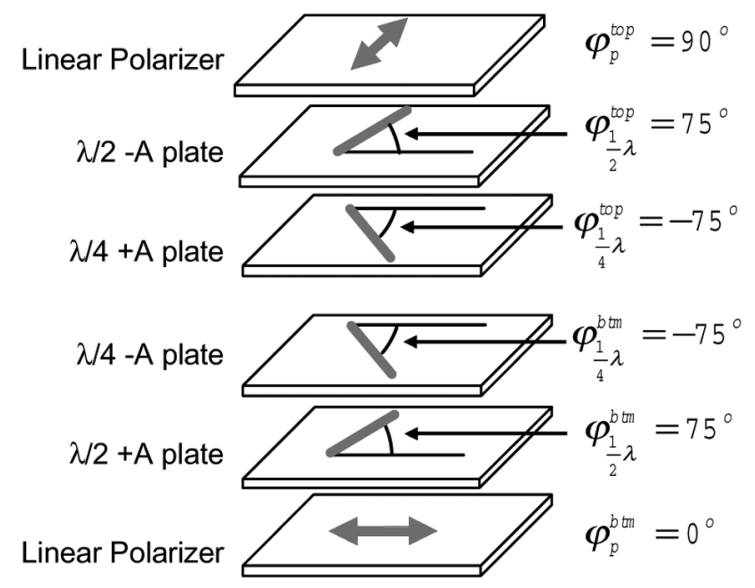

(a)

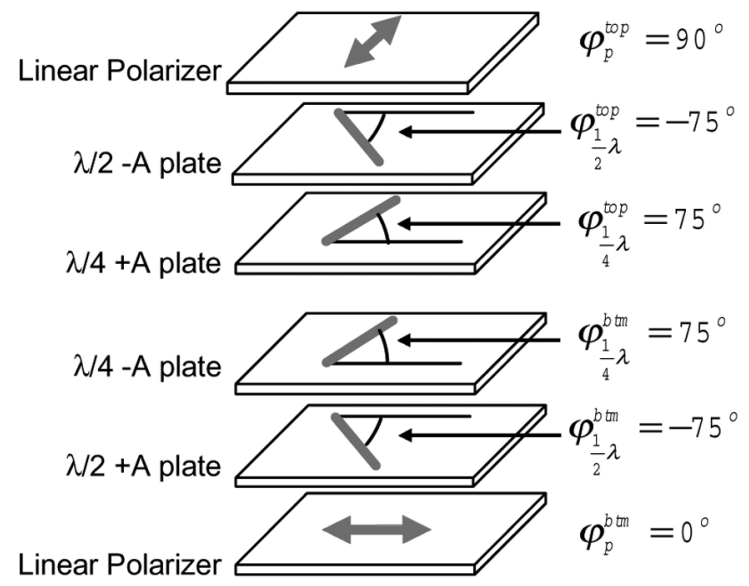

(b)

Fig. 6. Circular polarizer configuration with top half-wave plate having its optic axis at (a) $-15^{\circ}$ and (b) $15^{\circ}$ away from the transmission axis of the top linear polarizer.

\section{RESULTS}

\section{A. Spectral Bandwidth and Off-Axis Light Leakage}

Fig. 7 shows the simulated wavelength-dependent light leakage ( $\mathrm{T}$ or $\mathrm{R}$ ) for configuration in Fig. 6(a) at normal and off-axis viewing directions. The spectral $\mathrm{T}$ and $\mathrm{R}$ in the new design at normal incidence are quite similar to those in the conventional circular polarizers as shown in Fig. 2(d), but their mechanisms to maintain a broadband operation are different. For the transmissive mode, in the conventional design, the broad bandwidth is achieved by setting the top and bottom wave plates (half-wave or quarter-wave) orthogonal to each other. But in the new configuration, positive and negative A-plates are aligned parallel to compensate each other.

The difference in their off-axis performance is quite evident. The new design in Fig. 6(a) using opposite birefringence A-plates has much better off-axis compensation. The light leakages viewed at polar angle $\theta_{\text {inc }}=80^{\circ}$ and azimuthal angle $\varphi_{\text {inc }}=0^{\circ}$ are almost identical to the one from the normal direction at $\theta_{\text {inc }}=0^{\circ}$. The conventional circular polarizer, on the other hand, produces a $\sim 5 \%$ light leakage in the whole visible spectrum, when viewed even from $\theta_{\text {inc }}=40^{\circ}$. For reflective mode, the new design shows a lower light leakage in most of

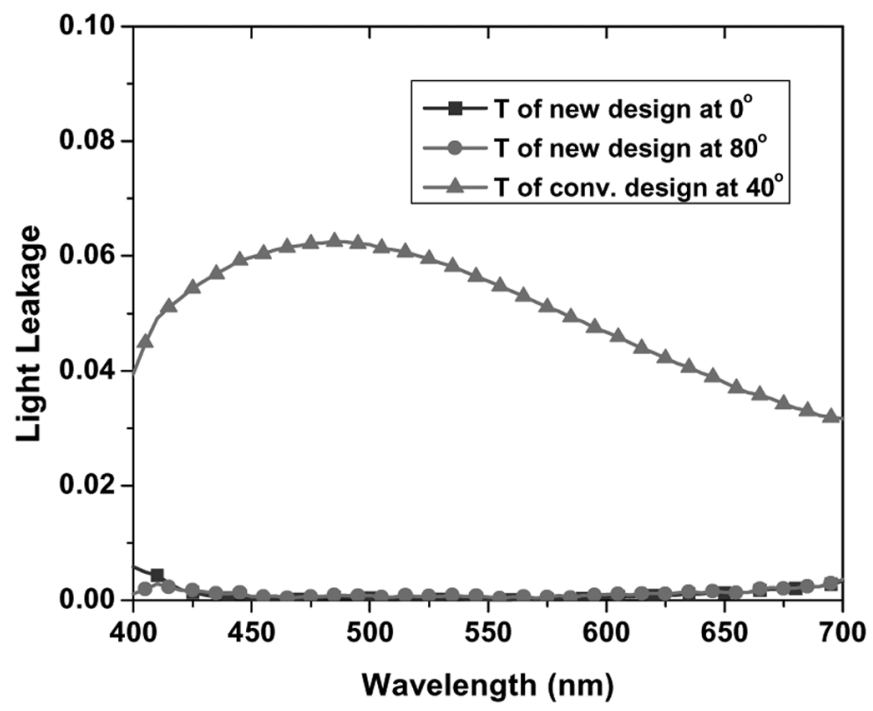

(a)

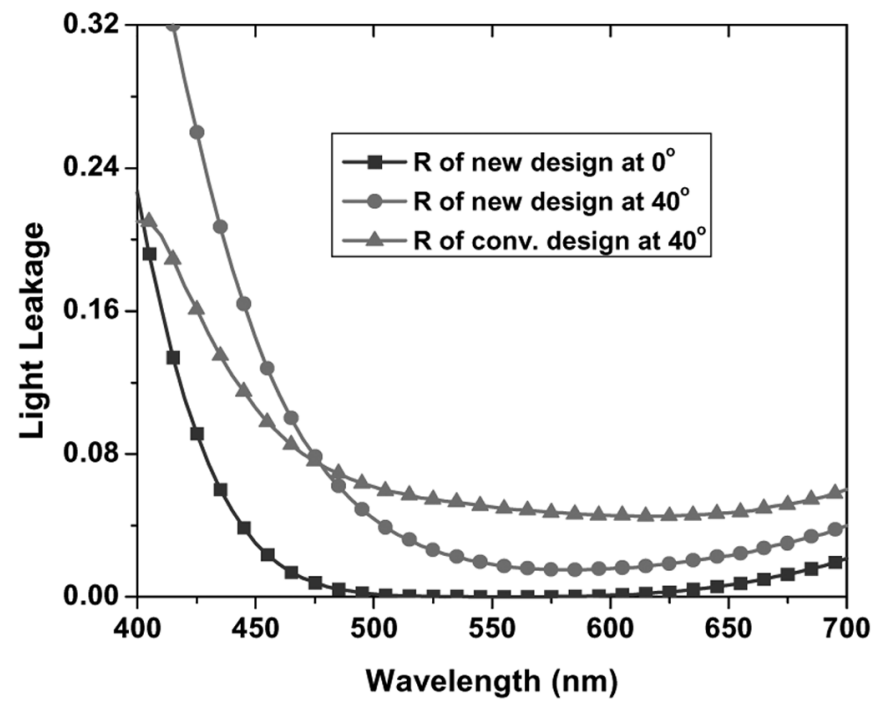

(b)

Fig. 7. Spectral light leakage from (a) the T-mode and (b) the R-mode when viewed at $\varphi_{\text {inc }}=0^{\circ}$.

the visible spectrum than the conventional one when viewed at $\theta_{\text {inc }}=40^{\circ}$, as shown in Fig. 7(b). The self-compensation from the opposite birefringence between the top half-wave and quarter-wave plates is inadequate to cancel each other as that for the transmissive mode, which can be clearly seen from Fig. 7(a) and (b).

Fig. 8(a) shows the polarization trace for the transmissive mode on the Poincaré sphere when viewed at $\theta_{\text {inc }}=70^{\circ}$ and $\varphi_{\text {inc }}=-45^{\circ}$. It demonstrates a self-compensation by opposite birefringence in the uniaxial A-plates, indicating by the fact the final polarization state $\mathbf{F}$ goes back to the initial polarization state at point $\mathbf{T}$. Consequently, the off-axis light leakage only results from the disparity between the top and the bottom polarizers viewed from an oblique angle. As shown in Fig. 8(b), the maximum light leakage mainly occurs at the bisector direction at $\varphi_{\text {inc }} \sim \pm 45^{\circ}$ and $\pm 135^{\circ}$. Over the entire viewing cone, the maximum light leakage from two crossed circular polarizers is 


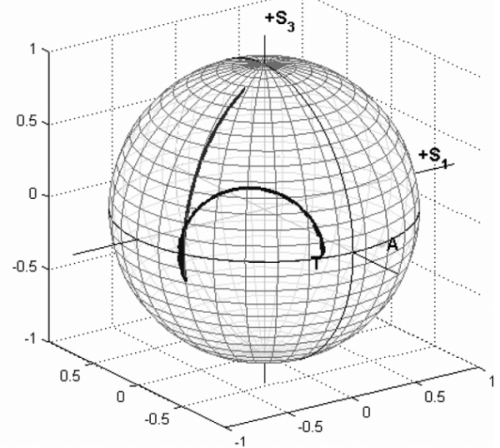

(a)

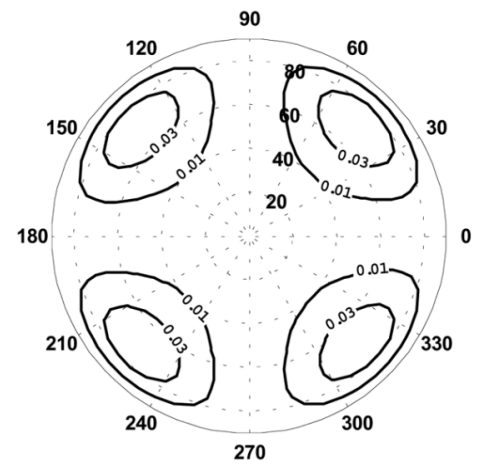

(b)

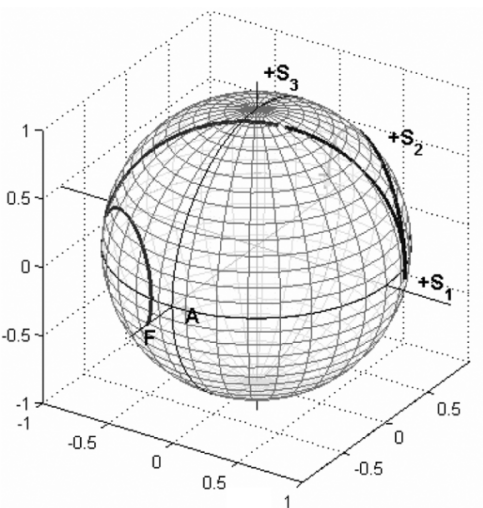

(c)

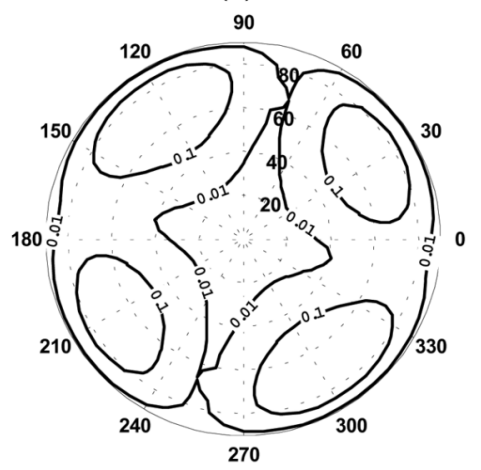

(d)

Fig. 8. (a) Polarization trace when viewed at $\theta_{\text {inc }}=70^{\circ}$ and $\varphi_{\text {inc }}=-45^{\circ}$ for the T-mode and (b) its angular light leakage; (c) polarization trace when viewed at $\theta_{\text {inc }}=40^{\circ}$ and $\varphi_{\text {inc }}=-45^{\circ}$ for the R-mode; and (d) its angular light leakage.

suppressed to below $\sim 3.5 \%$ which is much better than that in the conventional circular polarizers $\left(10 \%\right.$ at about $\left.40^{\circ}\right)$.

Fig. 8(c) shows the polarization trace for the reflective mode when viewed $\theta_{\text {inc }}=40^{\circ}$ and $\varphi_{\text {inc }}=-45^{\circ}$. As compared to that in Fig. 3(c), the final polarization at point $\mathbf{F}$ is closer to the absorption direction at point $\mathbf{A}$, which results from the fact that half-wave and quarter-wave plates with opposite birefringence make the polarization undergo a different trace than the conventional one in Fig. 3(c). Consequently, the light leakage shown in Fig. 8(d) from the top circular polarizer is also reduced as compared to that in Fig. 3(d).

Nevertheless, the off-axis light leakage can be further reduced by optimizing the film parameters. Detailed discussion is given in Section III-B for a case study using the designed circular polarizers in conjunction with a normally black VA cell. Here the residual phase retardation from the negative $\mathrm{C}$-plate and the LC layer (like a positive C-plate) can be taken as an additional freedom in optimization.

\section{B. Viewing Angle Including a Liquid Crystal Cell}

The aforementioned circular polarizer configuration is further applied to a MVA transflective LCD to verify our theory. By adjusting the film parameters, we obtained an optimum optical configuration, as shown in Fig. 9(a), where a negative C-plate is employed to compensate the LC cell at oblique angles [22]. Here Merck MLC-6608 with ordinary and extraordinary refractive indices $n_{\circ}=1.4789$ and $n_{e}=1.5622$ at $\lambda=546 \mathrm{~nm}$ [23] is employed and the cell gap is set at $4 \mu \mathrm{m}$. The negative C-plate with its refractive indices $n_{e}=1.4929$ and $n_{\circ}=$ 1.5028 at $\lambda=546 \mathrm{~nm}$ is designed with a phase retardation $d \Delta n \sim-365 \mathrm{~nm}$ at $\lambda=550 \mathrm{~nm}$. The phase retardation of the half-wave and quarter-wave plates at $\lambda=550 \mathrm{~nm}$ are designed at 235 and $117.5 \mathrm{~nm}$, respectively. Also, the optic axes of the top and bottom half-wave plates are aligned $\pm 2^{\circ}$ apart from their initial values, while two orientation angles still satisfy (4) within each circular polarizer. Under such a configuration, the overall light leakages in Fig. 8(b) and (d) can be greatly suppressed. Accordingly, for the transmissive mode, as shown in Fig. 9(b), a light leakage less than $\sim 1.5 \times 10^{-2}$ is obtained throughout the entire viewing cone. For the reflective mode as shown in Fig. 9(c), the maximum light leakage is $\sim 0.16$, but its central light leakage within $40^{\circ}$ is still quite small.

The compensation mechanism involved in Fig. 9(a) for achieving a small global light leakage is illustrated by Fig. 10(a) and 10(b), where the polarization of the incident light is traced on the Poincaré sphere when viewed at both $\left(\theta_{\text {inc }}=70^{\circ}\right.$, $\left.\varphi_{\text {inc }}=-45^{\circ}\right)$ and $\left(\theta_{\text {inc }}=70^{\circ}, \varphi_{\text {inc }}=0^{\circ}\right)$. In Fig. 10(a), at the bisector direction with $\varphi_{\text {inc }}=-45^{\circ}$, the absorption axis of the top polarizer (at point $\mathbf{A}$ ) and transmission axis of the bottom polarizer (at point $\mathbf{T}$ ) departs from each other. The incident light from the bottom linear polarizer is first moved from point $\mathbf{T}$ to point $\mathbf{B}$ by the bottom half-wave and quarter-wave plates. After passing the LC cell which performs like a positive C-plate, it is converted to point $\mathbf{C}$. The following negative C-plate moves it to point $\mathbf{D}$ in an opposite direction, which is intentionally designed to be away from point B. Subsequently, the top quarter-wave plate and half-wave plates further move the light from point $\mathbf{D}$ to point $\mathbf{F}$, which is much closer to point $\mathbf{A}$ than that in Fig. 8(a). On the other hand, when viewed at $\varphi_{\text {inc }}=0^{\circ}$ where point $\mathbf{T}$ and point $\mathbf{A}$ overlap with each regardless of the value of $\theta_{\text {inc }}$, the separation between points $\mathbf{B}$ and $\mathbf{D}$ makes the final polarization at point $\mathbf{F}$ away from point $\mathbf{A}$ (however, if $\mathbf{B}$ and $\mathbf{D}$ overlaps at $\varphi_{\text {inc }}=0^{\circ}$, final 


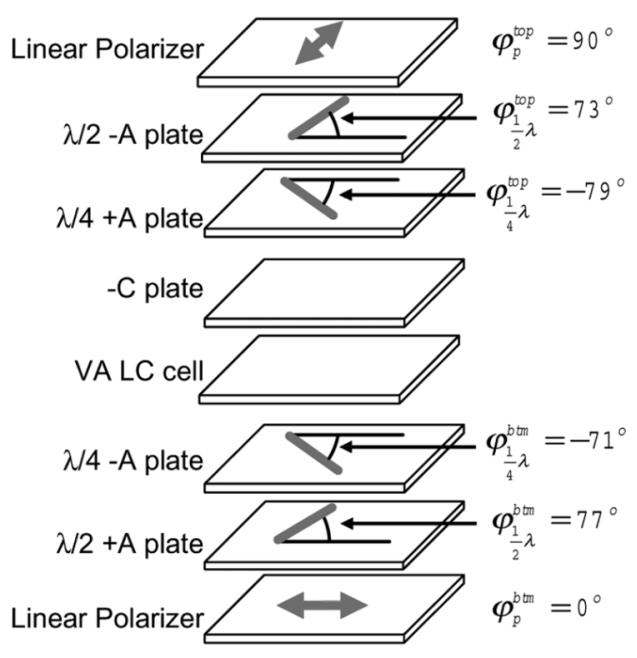

(a)

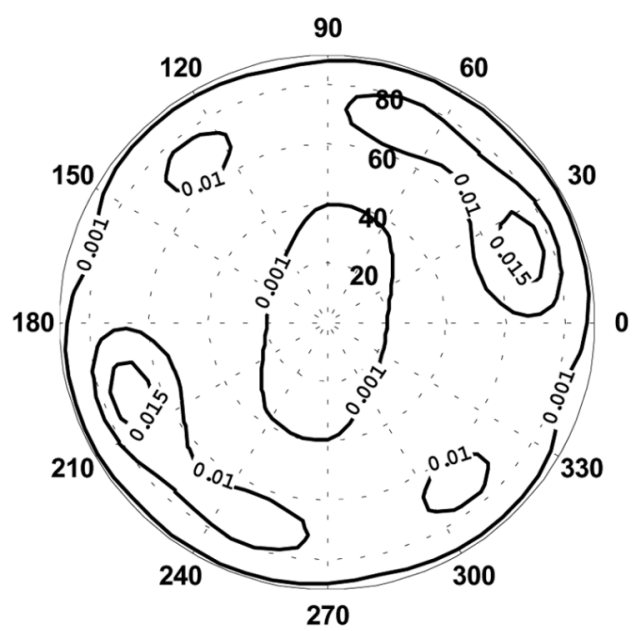

(b)

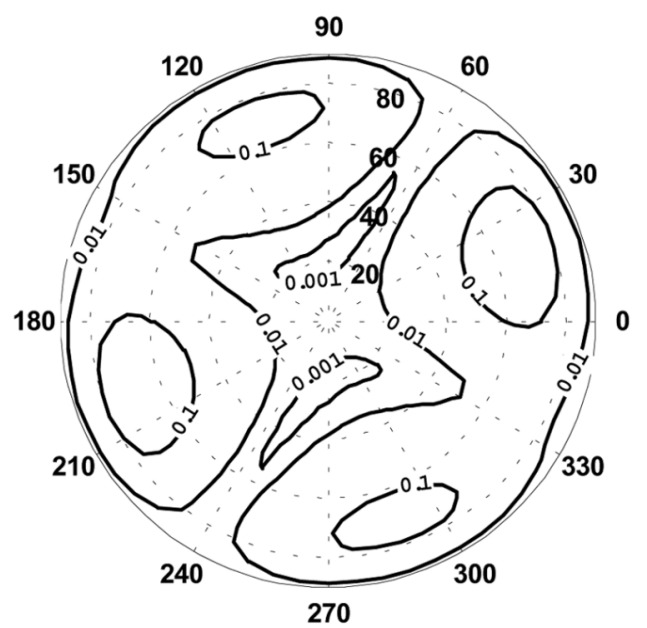

(c)

Fig. 9. (a) Film configuration of the optimized VA transflective LCD; light leakages from (b) T-mode and (c) R-mode.

polarization $\mathbf{F}$ will go back to point $\mathbf{A}$.) In other words, the compensations at these two directions contradict each other, and a tradeoff for global minimization needs to be considered, yielding the optimum configuration in Fig. 9(a).

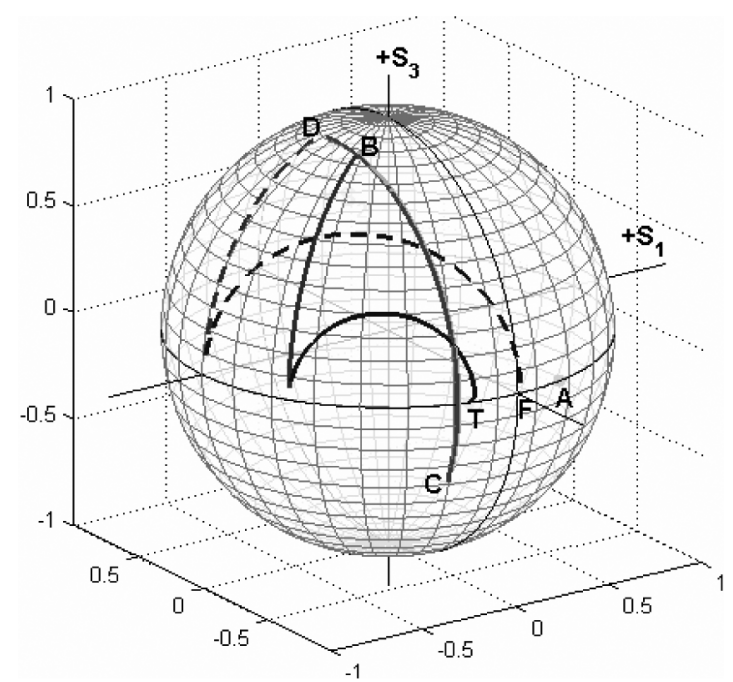

(a)

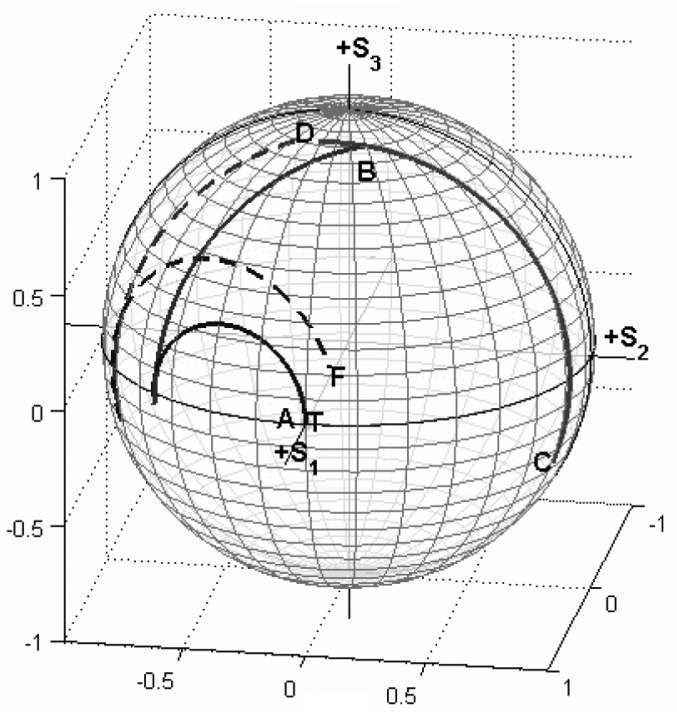

(b)

Fig. 10. Polarization trace on the Poincaré sphere at $\theta_{\text {inc }}=70^{\circ}$ when viewed at (a) $\varphi_{\text {inc }}=-45^{\circ}$ and (b) $\varphi_{\text {inc }}=0^{\circ}$.

The spectral light leakages from above-mentioned cell configurations at dark state are shown in Fig. 11. The transmissive mode shows a broadband operation at both normal and off-axis incidences, which improves a lot as compared to the conventional broadband circular polarizers. For the reflective mode, it maintains a small light leakage at a broad bandwidth at the normal incidence and an increased reflectance at a large off-axis angle, which is similar to conventional broadband circular polarizers, but gains a big improvement as compared to the case using a linear polarizer with a single monochromatic quarter-wave plate. In addition, as the phase retardation value is set less than the central $550 \mathrm{~nm}$, the reflectance shifts towards the shorter wavelength region.

Fig. 12(a) and (b) shows the iso-contrast plots for the transmissive mode and reflective mode, respectively. With a decreased global light leakage, the viewing angle of a transmissive two-domain VA cell has its 10:1 contrast ratio expanded over the entire viewing cone and the 10:1 contrast ratio for 


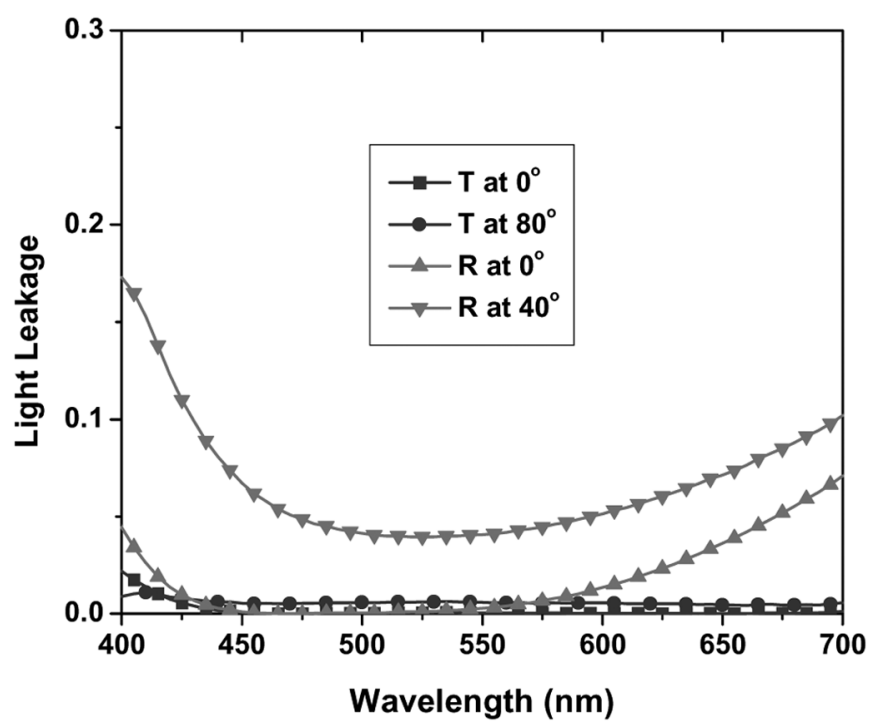

Fig. 11. Spectral light leakages from transflective LCD structure in Fig. 9(a).

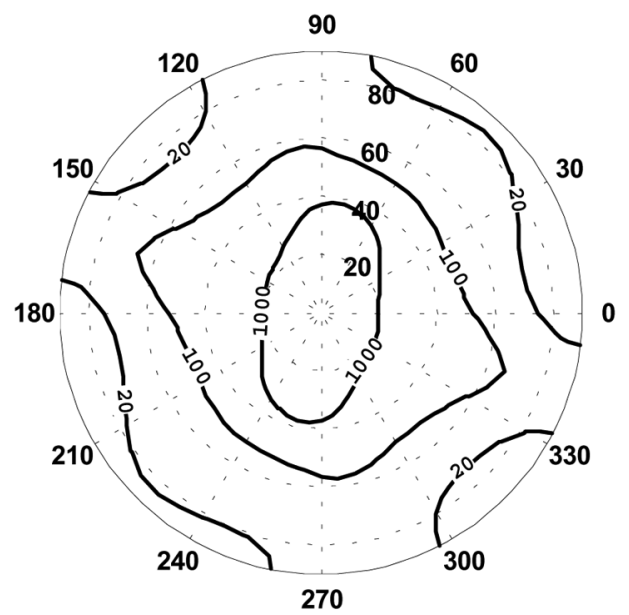

(a)

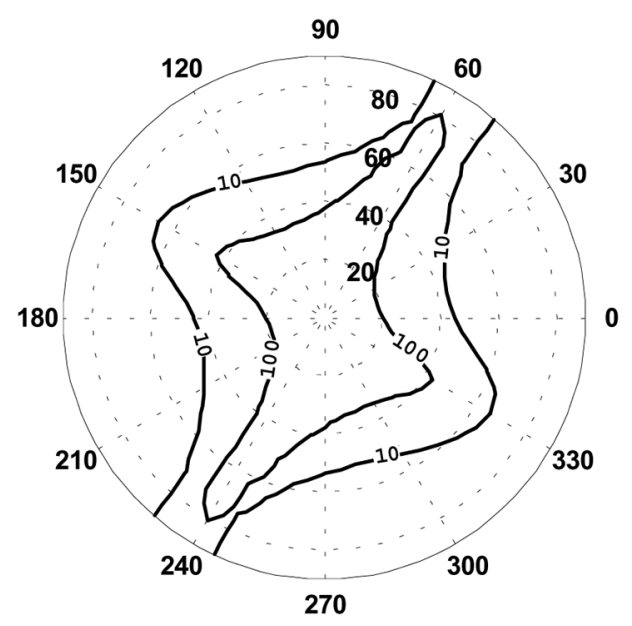

(b)

Fig. 12. Iso-contrast plots for (a) T-mode at $\lambda=550 \mathrm{~nm}$ and (b) of R-mode at $\lambda=550 \mathrm{~nm}$.

the reflective mode is achieved over $40^{\circ}$. Without introducing additional uniaxial films (two uniaxial films in each broadband circular polarizer) or biaxial films, this design greatly enhances the viewing angle of a transflective MVA LCD while maintaining a broadband operation. This configuration produces further possibility for MVA technology to be applied in high-end mobile displays.

\section{CONCLUSION}

We propose a simple and cost-effective circular polarizer configuration to achieve wide viewing angle and broad bandwidth for transflective liquid crystal displays. The alternating positive and negative birefringence uniaxial A-plates within the halfwave plate pair and the quarter-wave plate pair help to compensate the off-axis phase retardation. From our analysis, a normally black VA transflective LCD using our design could suppress the off-axis light leakage less than $1.5 \times 10^{-2}$ and obtain a contrast ratio over 10:1 throughout the entire viewing cone. At the same time, we also obtained a broadband reflective mode from the same configuration, while its contrast ratio over 10:1 is expanded over $40^{\circ}$. Besides, we also found that for viewing angle optimization under circular polarizers, it requires to take the compensation into consideration at both the bisector direction and polarizer $T$-axis direction, which is quite different from that under two linear polarizers. We believe this circular polarizer will have a foreseeable impact on the transflective LCD technologies.

\section{REFERENCES}

[1] S. T. Wu and D. Y. Yang, Reflective Liquid Crystal Displays. Hoboken, NJ: Wiley, 2001.

[2] X. Zhu, Z. Ge, T. X. Wu, and S. T. Wu, "Transflective liquid crystal displays," J. Display Technol., vol. 1, pp. 15-29, 2005.

[3] H. D. Liu and S. C. Lin, "A novel design wide view angle partially reflective super multi-domain homeotropically aligned LCD," Soc. Inf. Display Tech. Dig., vol. 33, pp. 558-561, 2002.

[4] C. R. Sheu, K. H. Liu, L. P. Hsin, Y. Y. Fan, I. J. Lin, C. C. Chen, B. C. Chang, C. Y. Chen, and Y. R. Shen, "A novel LTPS transflective TFT LCD driving by double gamma method," Soc. Inf. Display Tech. Dig., vol. 34, pp. 653-655, 2003.

[5] M. P. Hong, S. I. Kim, Y. C. Yang, K. Chung, H. W. Do, S. J. Park, C. G. Jhun, G. D. Lee, T. H. Yoon, and J. C. Kim, "Low-twist verticallyaligned transflective LCD," Soc. Inf. Display Tech. Dig., vol. 35, pp. 34-37, 2004.

[6] Z. Ge, X. Zhu, R. Lu, T. X. Wu, and S. T. Wu, "Transflective liquid crystal display using commonly biased reflectors," App. Phys. Lett. vol. 90, pp. 221111-221111, 2007.

[7] J. Tanno, M. Morimoto, K. Igeta, H. Imayama, S. Komura, and T. Nagata, "A new transflective IPS-LCD with high contrast ratio and wide viewing angle performance," in Conf. Proc. Int. Display Workshops, 2006, pp. 635-638

[8] H. Yoshida, Y. Tasaka, Y. Tanaka, H. Sukenori, Y. Koike, and K. Okamoto, "MVA LCD for notebook or mobile PCS with high transmittance, high contrast ratio, and wide angle viewing," Soc. Inf. Display Tech. Dig., vol. 35, pp. 6-9, 2004.

[9] S. Pancharatnam, "Achromatic combinations of birefringent plates," Proc. Indian Acad. Sci. A, vol. 41, pp. 130-144, 1956.

[10] T. H. Yoon, G. D. Lee, and J. C. Kim, "Nontwist quarter-wave liquid crystal cell for a high-contrast reflective display," Opt. Lett., vol. 25, pp. 1547-1549, 2000.

[11] T. Ishinabe, T. Miyashita, and T. Uchida, "Design of a quarter wave plate with wide viewing angle and wide wavelength range for high quality reflective LCDs," Soc. Inf. Display Tech. Dig., vol. 32, pp. 906-909, 2001.

[12] H. Yoshimi, S. Yano, and Y. Fujimura, "Optical films for reflective LCDs to achieve high image quality," Soc. Inf. Display Tech. Dig., vol. 33 , pp. 862-865, 2002.

[13] Q. Hong, T. X. Wu, X. Zhu, R. Lu, and S. T. Wu, "Designs of wide-view and broadband circular polarizers," Opt. Expr., vol. 13, pp. 8318-8331, 2005.

[14] Q. Hong, T. X. Wu, R. Lu, and S. T. Wu, "Wide-view circular polarizer consisting of a linear polarizer and two biaxial films," Opt. Expr., vol. 13, pp. 10777-10783, 2005.

[15] C. H. Lin, "Extraordinarily wide-view and high-transmittance vertically aligned liquid crystal displays," Appl. Phys. Lett., vol. 90, pp. 151112-151112, 2007. 
[16] S. Huard, Polarization of Light. New York: Wiley, 1997.

[17] X. Zhu, Z. Ge, and S. T. Wu, "Analytical solutions for uniaxial-filmcompensated wide-view liquid crystal displays," J. Display Technol., vol. 2, pp. 2-20, 2006.

[18] A. Lien, "Extended jones matrix representation for the twisted nematic liquid-crystal display at oblique incidence," Appl. Phys. Lett., vol. 57, pp. $2767-2769,1990$.

[19] Z. Ge, T. X. Wu, X. Zhu, and S. T. Wu, "Reflective liquid crystal displays with asymmetric incidence and exit angles," J. Opt. Soc. Amer. A, vol. 22, pp. 966-977, 2005.

[20] J. Li and S. T. Wu, "Extended Cauchy equations for the refractive indices of liquid crystals," J. Appl. Phys., vol. 95, pp. 896-901, 2004.

[21] S. T. Wu and C. S. Wu, "Mixed-mode twisted nematic liquid crystal cells for reflective displays," Appl. Phys. Lett., vol. 68, pp. 1455-1457, 1996.

[22] J. Chen, K. H. Kim, J. J. Jyu, J. H. Souk, J. R. Kelly, and P. J. Bos, "Optimum film compensation modes for TN and VA LCDs," Soc. Inf. Display Tech. Dig., vol. 29, pp. 315-318, 1998.

[23] J. Li, C. H. Wen, S. Gauza, R. Lu, and S. T. Wu, "Refractive indices of liquid crystals for display applications," J. Display Technol., vol. 1, no. 1, pp. 51-61, Sep. 2005.

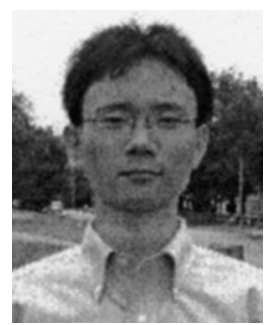

Zhibing Ge (S'02) received the B.S. and M.S. degrees in electrical engineering from Zhejiang University, China, in 2002, and 2004, respectively. He is currently working toward the $\mathrm{Ph}$. D. degree in the area of liquid crystal display modeling and application.

$\mathrm{He}$ joined the Electrical and Computer Engineering Department, University of Central Florida, Orlando, as a graduate student in 2002, and his current research interests include transflective liquid crystal displays and numerical methods in modeling of liquid crystal devices.

Meizi Jiao received the B.S. degree in optics from Zhejiang University (ZJU) in 2005 , and is currently working toward the Ph.D. degree at the College of Optics and Photonics, University of Central Florida, Orlando.

Her currently research interests include developing transflective LCDs and optical compensation films development for LCD applications.

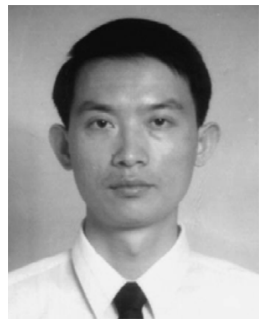

Ruibo Lu received the Ph.D. degree in optics from Department of Physics, Fudan University, Shanghai, China, in 1998, and M.S. degree in applied physics from Department of Physics, East China University of Science and Technology, Shanghai, China, in 1995. His research work in Ph.D. focused on liquid crystal alignment and ferroelectric liquid crystal devices for display and advanced optical applications.

$\mathrm{He}$ was a faculty member in the Department of Physics, and later in the Department of Optical Science and Engineering, Fudan University, Shanghai, China, from 1998 to 2001. He was an optical engineer in Lightwaves2020 Inc., San Jose, CA, from 2001 to 2002. Since then, he joined the School of Optics/ CREOL (now as College of Optics and Photonics), University of Central Florida, Orlando, as a research scientist. His research interests include liquid crystal display technology, wide viewing angle for liquid crystal TVs, liquid crystal components for optical communications and optical imaging using liquid crystal medium.

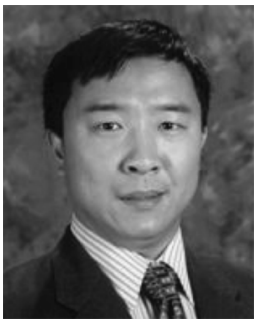

Thomas X. Wu (S'96-M'98-SM'02) received the M.S. and Ph.D. degrees in electrical engineering from the University of Pennsylvania in 1997 and 1999.

In the fall of 1999, he joined the School of Electrical Engineering and Computer Science, University of Central Florida (UCF) as an assistant professor, and in 2005, was promoted to associate professor, with tenure. His current research interests include $\mathrm{RF}$ integrated circuits and packaging, electrical machinery and magnetic device, liquid crystal device, computational physics, and nano electronics.

Prof. Wu was chairman of IEEE Orlando Section in 2004, and chairman of IEEE MTT and AP Joint Chapter from 2003 to 2004. He was listed in Whos Who in Science and Engineering, Whos Who in America, and Whos Who in the world. He was awarded Distinguished Researcher of the Department of Electrical and Computer Engineering in 2003, Distinguished Researcher of College of Engineering and Computer Science in 2004, and University Research Incentive Award in 2005. He was also awarded Excellence for Undergraduate Teaching Award from the School of Electrical Engineering and Computer Science in January 2006, and Excellence for Undergraduate Teaching Award from the College of Engineering and Computer Science in February 2006.

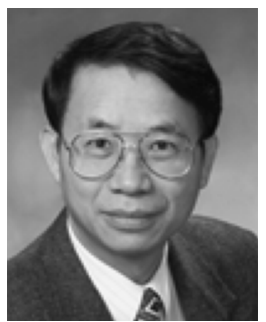

Shin-Tson Wu (M'98-SM'99-F'04) received the B.S. degree in physics from National Taiwan University, and the Ph.D. degree from the University of Southern California, Los Angeles.

$\mathrm{He}$ is a PREP professor at College of Optics and Photonics, University of Central Florida (UCF). His studies at UCF concentrate in foveated imaging, bio-photonics, optical communications, liquid crystal displays, and liquid crystal materials. Prior to joining UCF in 2001, he worked at Hughes Research Laboratories, Malibu, CA, for 18 years. He has co-authored 4 books: Fundamentals of Liquid Crystal Devices (Wiley, 2006, with D. K. Yang); Introduction to Microdisplays (Wiley, 2006, with D. Armitage and I. Underwood) Reflective Liquid Crystal Displays (Wiley, 2001, with D. K. Yang) and Optics and Nonlinear Optics of Liquid Crystals (World Scientific, 1993, with L. C. Khoo), 5 book chapters, and over 350 journal papers. He has more than 55 issued and pending patents. Several of his patents have been implemented in display and photonic devices.

Dr. Wu is a Fellow of the Society of Information Display (SID) and Optical Society of America (OSA).

Wang-Yang $\mathbf{L i}$ received the Ph.D. degree in electro-optical engineering from National Chiao Tung University, Taiwan, R.O.C., in 1998.

He joined Chi-Mei Optoelectronics (CMO), Tainan, Taiwan, R.O.C., in 1999 as a R\&D engineer. From 1999 to 2002, he led a team to improve the optical performance of LCD modules for notebooks and desktop monitors. Later, he became a manager of the R\&D group in 2002. Presently, he is the manager of the CMO LCD TV Head Division.

Chung-Kuang Wei received the Ph.D. degree in electro-optical engineering from National Chiao Tung University, Taiwan, R.O.C., in 1992.

He presently is R\& D manager of the LCD Head Division, Chi-Mei Optoelectronics (CMO), Tainan, Taiwan, R.O.C., 